Editorial

\title{
The eye in AI: artificial intelligence in ophthalmology
}

Stuart Keel PhD FRANZCO ${ }^{1,2}$ and Peter van Wijngaarden PhD FRANZCO ${ }^{1,2}$

1. Centre for Eye Research Australia, Royal Victorian Eye and Ear Hospital, Melbourne, Australia.

2. Ophthalmology, University of Melbourne, Department of Surgery, Melbourne, Australia.

Conflict of interest: None

Funding sources: None

This is the author manuscript accepted for publication and has undergone full peer review but has not been through the copyediting, typesetting, pagination and proofreading process, which may lead to differences between this version and the Version of Record. Please cite this article as doi: $10.1111 /$ ceo.13435

This article is protected by copyright. All rights reserved. 
The convergence of major developments in artificial intelligence (AI) for image analysis with advances in clinical imaging technologies has major implications for the practice of medicine. Gains in AI system performance have been the product of improvements in computing hardware and progress in algorithm design, such that large volumes of data can now be processed with great accuracy at extraordinary speeds. As Hogarty and colleagues illustrate in this edition of the Journal, the discipline of ophthalmology is at the forefront of the AI revolution, with a growing body of research indicating that AI systems can be applied to a wide range of ophthalmic imaging methods across a broad range of disease categories with remarkable performance. ${ }^{1}$

As a testament to rapid advancements in this field, several important publications have emerged since Hogarty and colleagues undertook their literature search. Grassmann et al. developed a deep learning algorithm on 86,770 manually graded color fundus images from the Age-Related Eye Disease Study (AREDS) database and achieved a high overall accuracy for the classification of age-related macular degeneration (AMD) in an independent validation dataset (overall accuracy for early or late $A M D=84.2 \%) .{ }^{2}$ In addition, Li et al. reported the successful application of a deep learning algorithm for discrimination of color fundus photographs with referable glaucomatous optic neuropathy (suspect or certain) from non-referable (unlikely) photos. $^{3}$

Another recent publication with profound implications for the implementation of AI systems in the clinic describes the evaluation of a deep learning system for the detection of referable diabetic retinopathy (>mild NPDR and/or DME). ${ }^{4}$ The trial included 900 participants with diabetes who were recruited from 10 primary care practices in the United States. Participants first underwent conventional two-field retinal photography (non-mydriatic unless an AI image quality assessment system 
deemed mydriasis necessary) and these images underwent AI system grading. Participants then underwent mydriatic wide-field stereoscopic fundus photography and macular OCT imaging for expert human grading to establish the ground truth for each participant, against which AI system performance was measured. Performance of the AI system exceeded predetermined standards (sensitivity $=87.2 \%(>85 \%)$; specificity $=90.7 \%(>82.5 \%)$ ). On the basis of these findings, the U.S. Food \& Drug Administration (FDA) has authorised the system for use by health professionals for the autonomous detection of diabetic retinopathy (>mild NPDR) and DME. This landmark approval is the first ever FDA-authorized AI diagnostic system in any field of medicine.

Arguably the most significant breakthrough in the field to date has arisen from a collaboration between DeepMind, leaders in the development of AI systems, and a team of clinicians and academics from Moorfields Eye Hospital and University College London. ${ }^{5}$ An AI system was trained on 14,884 OCT scans to detect more than 50 common retinal diagnoses, representing a wide variety of conditions affecting patients attending a tertiary referral eye hospital. An independent sample of images from 997 patients with gold-standard labels based on an expert panel decision, using actual clinical outcome data, formed the validation dataset. AI system performance on the validation dataset was compared against 4 retinal specialists and 4 optometrists from Moorfields Eye Hospital. Of note, the AI classification was based on the OCT scan alone, while human graders had acess to OCT scans, fundus images and medical history data. The AI system demonstrated performance reaching or exceeding that of human graders (error rate: $\mathrm{AI}=5.5 \%$ vs. experts, range $=5.5 \%-13.1 \%$ ) for a challenging multi-class decision problem (4 referral categories: urgent, semi-urgent, routine, observation only). Importantly, system performance was robust when images from 2 different OCT devices were used. This 
study indicates sub-specialist expert level performance of an OCT AI system with clinically-relevant stratification of referral urgency.

Despite enormous promise, the potential of this technology must be viewed in light of its application in real-world clinical settings. Until recently, the basis for the AI system classification of a given image has been inscrutable to clinicians. Several recent studies report the development of visualization tools that highlight the regions of a given image that are most contributory to the final AI classification. ${ }^{5,6}$ The routine deployment of such tools is likely to help promote clinician and patient acceptance of AI image analysis systems. As Hogarty and colleagues note, the broader clinical applicability of a given AI image analysis study should be informed by the representativeness of the training and validation datasets. Training datasets need to be of adequate size to include a wide range of disease and non-disease variables and be representative of a wide range of ethnicities, imaging devices and protocols for external applicability and to minimise scope for bias. AI systems may be validated using an independent external dataset (gold standard) or via internal hold-out methods, where a proportion of the training dataset is set aside for testing purposes. The latter approach may be more prone to under-fitting, where important predictors are missing, or over-fitting, where the prediction model is too closely adapted to the dataset on which it was trained, at the expense of being poorly predictive of other datasets.

The timely review by Hogarty and colleagues highlights the major innovations that are being made in the application of AI in ophthalmology. It behoves eye health care providers to remain abreast of developments in this field and to collectively inform the manner in which these systems are incorporated in clinical practice. The potential for these systems to improve patient access to timely and accurate diagnosis and clinical risk stratification needs to be tempered by risk controls around privacy and data security, as well as the need for ongoing human input into 
diagnosis and clinical decision making that presently lies at the centre of the doctorpatient relationship and which remains the foundation of health care.

\section{REFERENCES}

1. Hogarty DT, Mackey DA, Hewitt AW. Current state and future prospects of artificial intelligence in ophthalmology: a review. Clinical \& experimental ophthalmology 2018.

2. Grassmann F, Mengelkamp J, Brandl C, et al. A Deep Learning Algorithm for Prediction of Age-Related Eye Disease Study Severity Scale for Age-Related Macular Degeneration from Color Fundus Photography. Ophthalmology 2018. 125(9); 14101420

3. Li Z, He Y, Keel S, Meng W, Chang RT, He M. Efficacy of a Deep Learning System for Detecting Glaucomatous Optic Neuropathy Based on Color Fundus Photographs. Ophthalmology 2018; 125(8): 1199-206.

4. Abramoff MD, Lavin PT, Birch $M$, Shah NH, Folk JC. Pivotal trial of an autonomous AI-based diagnostic system for detection of diabetic retinopathy in primary care offices. NPJ Digital Medicine 2018; 1(39).

5. De Fauw J, Ledsam JR, Romera-Paredes B, et al. Clinically applicable deep learning for diagnosis and referral in retinal disease. Nature medicine 2018; 24(9): 1342-50.

6. Gargeya R, Leng T. Automated Identification of Diabetic Retinopathy Using Deep Learning. Ophthalmology 2017; 124(7): 962-9. 


\section{University Library}

\section{- M M I E E R VA A gateway to Melbourne's research publications}

Minerva Access is the Institutional Repository of The University of Melbourne

Author/s:

Keel, S; van Wijngaarden, $\mathrm{P}$

Title:

The eye in Al: artificial intelligence in ophthalmology

Date:

2019-01-01

Citation:

Keel, S. \& van Wijngaarden, P. (2019). The eye in Al: artificial intelligence in ophthalmology. CLINICAL AND EXPERIMENTAL OPHTHALMOLOGY, 47 (1), pp.5-6. https://doi.org/10.1111/ ceo.13435.

Persistent Link:

http://hdl.handle.net/11343/284841 